\title{
Questions EMERGE as Biogen claim aducanumab turnaround
}

Having shelved aducanumab in Alzheimer's disease following Phase 3 futility, Biogen now say a more complete analysis indicates efficacy with longer exposure to higher doses and plan submission for FDA approval. Major unanswered questions arise from the trials and the limited data released so far do not establish efficacy.

Biogen's aducanumab is a human immunoglobulin G1 monoclonal antibody with high affinity for a conformational epitope on aggregated forms of amyloid beta (A $\beta$ ). Phase 1 trial data, showing treatment removed $A \beta$ from the brain and potentially reduced cognitive decline, were published in Nature in 2016 ${ }^{1}$. Two identical Phase 3 trials, ENGAGE (started August 2015) and EMERGE (started September 2015), each enrolled over 1600 participants with early Alzheimer's disease (AD). The trials were discontinued in March 2019, when futility analyses based on data from around half the enrolled participants who had completed 18 months' follow up by December 2018 indicated little chance of efficacy $^{2}$. In April 2019, Biogen shelved a planned Phase 3 secondary AD prevention trial ${ }^{3}$ and it seemed this was case closed on yet another promising contender to be the first effective Alzheimermodifier. On 22 October 2019, however, Biogen presented an analysis based on more complete ENGAGE and EMERGE data. In a slide presentation for investors, headlined: "Sufficient exposure to high dose aducanumab reduced clinical decline across multiple clinical endpoints", the company reported that they were making a submission for United States Food and Drug Administration (FDA) approval ${ }^{4}$.

Encouragingly, if you suspend recollection of the futility analysis, information from EMERGE looks positive. It's important to point out that Biogen have only revealed percentage differences on outcome measures between drug and placebo groups with accompanying p-values. We aren't therefore looking at actual participant data when we are told that, compared with placebo, high dose participants in EMERGE experienced a 23\% reduction in decline on the Clinical Dementia Rating-Sum of Boxes (CDR-SB), along with a $27 \%$ and $40 \%$ reduction on the AD Assessment Scale-Cognitive Subscale 13 Items (ADAS-Cog 13) and AD Cooperative Study-Activities of Daily Living Inventory Mild Cognitive Impairment Version (ADCS-ADL-MCI) respectively. Although it isn't possible to calculate treatment effect sizes from such limited reporting, Biogen's announcement should have been a cause for celebration for everyone, except perhaps the unfortunate architect of the (also) unreleased futility analysis. How happy too, that Biogen already had a completed identical trial to replicate their findings. 
Cruelly, ENGAGE turns out to have been EMERGE's evil trial twin and no outcomes were significant or even showed hints of efficacy.

Biogen have tried to explain the futility analysis and what is essentially a failed replication. They claim that at the time of the futility analysis, EMERGE was actually "trending positive", while ENGAGE was not, and that later data pushed EMERGE over the line into significance. Between December 2018 when data were cut for the futility analysis and March 2019 when the trials were discontinued, an additional 179 EMERGE and 139 ENGAGE participants completed 18 months' follow-up to make the final numbers 982 and 1084 respectively. Statistically, around a third of these additional participants would have been randomised to the high dose arms, representing $18 \%$ and $13 \%$ of the 340 and 345 high dose participants in EMERGE and ENGAGE who comprised what Biogen have called the 'opportunity to complete' population for their unplanned subgroup analyses. Either these participants in EMERGE showed very large treatment responses, or the last 60 randomised to placebo very large decline rates, in order to drive the overall group results from trending-positive-but-considered-futile to significantly positive. Without seeing the actual quantitative data we can't comment further. The replication failure is even harder to explain. Biogen suggest that differences between the trials arose from differential effects of two protocol amendments. These allowed participants who had been dosesuspended due to amyloid-related imaging abnormalities (ARIA) to resume their assigned dose and ApoE4 carriers to titrate to $10 \mathrm{mg} / \mathrm{kg}$. The amendments would have increased exposure of participants to higher antibody doses, but without seeing actual data, the argument that this would have differentially benefitted EMERGE because it started one month after ENGAGE doesn't convincingly explain the different results.

Positive treatment effects that appear in analyses of subsets of participants should always be received with caution. In the same therapeutic area, after failing to meet the primary efficacy endpoints for mild-moderate $A D$, secondary analyses restricted to mild $A D$ in the early solanezumab trials indicated $34 \%$ less cognitive and $18 \%$ less functional decline than with placebo, but there was not even a hint of efficacy in the moderate subgroup 5 . This led to EXPEDITION3, involving only mild participants, but which failed to show significant slowing of cognitive decline ${ }^{6}$. With a sufficiently large trial sample, even very small differences can be statistically significant ${ }^{7}$. If, and when, the full adacanumab data are made available, it will be essential to examine treatment effect sizes in order to judge whether any statistically significant differences between drug and placebo are actually clinically meaningful for $A D$ patients. The limited data released from Biogen includes two graphs ${ }^{4}$ showing change from baseline CDR-SB in a post-hoc and non-randomly selected compliance-based subset of patients who received $\geq 10$ uninterrupted high-dose aducanumab treatments. By visual inspection, the difference between this subgroup and all placebo participants at 18 months appears to be roughly 0.75 and 0.5 points in 
EMERGE and ENGAGE respectively (although with no accompanying p-values). A recent study estimated that the minimal clinically important treatment difference for CDR-SB should be 1 and 2 points for mild cognitive impairment and mild AD patients respectively ${ }^{8}$.

The advent of an effective treatment to slow AD progression would represent an important milestone. Biogen's claims will be welcomed as a confirmation for those scientists who have invested their careers in the amyloid hypothesis and have seen a string of treatment failures knock confidence in this. Biogen simply haven't released sufficient information to know if we should be celebrating what could be a Lazarus-like return for aducanumab, or whether the drug is a zombie, reanimated by Chance and selective and limited presentation of trial results. The Company have said that more information will be presented at the Clinical Trials on Alzheimer's disease (CTAD) meeting in December, but it is frustrating that commercial considerations have led to such incomplete and delayed reporting ${ }^{9}$. As we interpret this and any subsequent data reveals by the company that fall short of full disclosure, we should be mindful that Biogen will have had the results of all their analyses since June when they held an earlier meeting with the FDA. AD patients and the people who love and care for them are an important and potentially vulnerable group. Many of them will have assisted Biogen and other companies through their participation in clinical trials and they will receive news stories such as this latest example with mixed emotion, particularly after historical overselling of trial data from this area. Since Biogen's 22 October announcement, it has been very difficult to know what to say to the $A D$ community about the validity and clinical significance of the results and in particular whether the treatment, even if it receives regulatory approval, is likely to deliver worthwhile benefits or be cost-effective. There are strong arguments that Biogen should come clean and present the full analyses without further delays.

\section{References:}

1. Sevigny, J. et al. The antibody aducanumab reduces $A \beta$ plaques in Alzheimer's disease. Nature 537, 50-56 (2016).

2. Biogen and Eisai to Discontinue Phase 3 ENGAGE and EMERGE Trials of aducanumab in Alzheimer's Disease | Biogen. Biogen Available at: http://investors.biogen.com/news-releases/newsrelease-details/biogen-and-eisai-discontinue-phase-3-engage-and-emerge-trials. (Accessed: 28th October 2019)

3. Biogen Q1 2019 Earnings Presentation. http://investors.biogen.com/static-files/1e8f9954-58d441cf-a6d7-6626338656c3

4. Biogen Q3 2019 Earnings Presentation. Available at: http://investors.biogen.com/staticfiles/40565136-b61f-4473-9e58-9be769bbac6c. 
5. Siemers, E. R. et al. Phase 3 solanezumab trials: Secondary outcomes in mild Alzheimer's disease patients. Alzheimers. Dement. 12, 110-120 (2016).

6. Honig, L. S. et al. Trial of Solanezumab for Mild Dementia Due to Alzheimer's Disease. N. Engl. J. Med. 378, 321-330 (2018).

7. Thompson, B. Statistical Significance, Result Importance, and Result Generalizability: Three Noteworthy But Somewhat Different Issues. Measurement and Evaluation in Counseling and Development 22, 2-6 (1989).

8. Andrews, J. S. et al. Disease severity and minimal clinically important differences in clinical outcome assessments for Alzheimer's disease clinical trials. Alzheimers. Dement. 5, 354-363 (2019).

9. Biogen surges $42 \%$ after stunning reversal sees it revive plans for Alzheimer's treatment (BIIB). Business Insider 